\title{
Targeted disruption of an essential vertebrate gene: ASF/SF2 is required for cell viability
}

\author{
Jin Wang, Yoshio Takagaki, and James L. Manley \\ Department of Biological Sciences, Columbia University, New York, New York 10027 USA
}

\begin{abstract}
Alternative splicing factor/splicing factor 2 (ASF/SF2) is the prototype of a family of nuclear proteins highly conserved throughout metazoa, the SR (serine/arginine) proteins. Based largely on in vitro studies, SR proteins have been suggested to play important roles in constitutive and alternative splicing of pre-mRNAs. Here we describe the development of a genetic system employing the chicken B-cell line DT40 to study the function of ASF/SF2 in vivo. The high level of homologous recombination and rapid growth rate of these cells allowed us to show first that $A S F / S F 2$ is an essential gene, and then to perform targeted disruption of both ASF/SF2 alleles, by creating a cell line in which the only source of ASF/SF2 is a human cDNA controlled by a tetracycline (tet)-repressible promoter. We show that addition of tet to these cells results in rapid depletion of ASF/SF2, concomitant accumulation of incompletely processed pre-mRNA, and subsequent cell death. The tet-induced lethality could be rescued by plasmids expressing wild-type ASF/SF2, but not several mutant derivatives, or other SR proteins. Heterozygous cell lines overexpressing human ASF/SF2 displayed significant reductions of endogenous ASF/SF2 mRNA, suggesting that ASF/SF2 mRNA levels are controlled by an autoregulatory loop. This system provides a novel method for genetic analysis of factors that function in basic processes in vertebrate cells.
\end{abstract}

[Key Words: Pre-mRNA splicing; SR proteins; chicken DT40 cells]

Received July 25, 1996; revised version accepted August 19, 1996.

The factors involved in pre-mRNA splicing have been studied intensively in recent years. In addition to the well-characterized small nuclear ribonucleoprotein particles (snRNPs), a large number of additional factors, referred to collectively as non-snRNP proteins, are required to catalyze and/or regulate the process (for review, see Moore et al. 1993). Prominent among the nonsnRNP proteins is a family of related factors called SR proteins (Zahler et al. 1992; for review, see Fu 1995; Manley and Tacke 1996). Eight SR proteins have been described to date in mammalian systems. All contain either one or two RNP-type RNA binding domains (RBD) at their amino termini, and regions of varying lengths of arginine/serine dipeptide repeats (RS domain) at their carboxy termini. Although clear homologs to the SR proteins have not been described in yeast, the proteins are highly conserved throughout metazoa. For example, specific individual family members have been found to share $\sim 65 \%$ identity between flies and humans (e.g., Screaton et al. 1995), and over $98 \%$ identity between chicken and humans (Vellard et al. 1992; see below).

As a result of extensive in vitro studies, SR proteins have been postulated to play multiple roles in premRNA splicing. First, SR proteins are thought to be essential, or general, splicing factors, as they can reconstitute splicing of model pre-mRNAs when added to cyto- plasmic S100 extracts, which lack SR proteins but contain all other essential splicing factors (Krainer et al. 1990b, 1991; Ge et al. 1991; Fu and Maniatis 1992a; Kim et al. 1992; Zahler et al. 1992; Cavaloc et al. 1994; Screaton et al. 1995). Given that in general any single SR protein can function by itself to activate splicing in this assay, it may be that they are functionally redundant as essential splicing factors. Second, addition of excess SR proteins to splicing-competent nuclear extracts has been shown to alter, in a concentration-dependent manner, utilization of competing splice sites in several alternatively spliced pre-mRNAs (e.g., Ge and Manley 1990; Krainer et al. 1990a; Fu et al. 1992; Zahler et al. 1993). Although certain individual SR proteins again show very similar activities in this assay (e.g., Fu et al. 1992), evidence has also been presented that other SR proteins can differ in their selection of competing alternative splice sites (e.g., Kim et al. 1992; Zahler et al. 1993). Finally, SR proteins have been implicated in the activity of RNA elements known as splicing enhancers. These sequences are found frequently in exons, and are required for splicing of the upstream intron, which is usually typified by the presence of a weak $5^{\prime}$ or $3^{\prime}$ consensus splice site (e.g., Hoshijima et al. 1991; Tian and Maniatis 1992; Watakabe et al. 1993). In contrast to their role as essential splicing factors, it appears that the activity of SR proteins in 
the function of splicing enhancers is largely nonredundant (Sun et al. 1993; Tian and Maniatis 1993; Staknis and Reed 1994; Ramachatesingh et al. 1995; Tacke and Manley 1995), likely reflecting at least in part differences in sequence-specific RNA binding.

Additional in vitro studies have begun to shed light on how the SR proteins actually function in splicing. Two prototypical SR proteins, ASF/SF2 (Ge' et al. 1991; Krainer et al. 1991) and SC35 (Fu and Maniatis 1992b), have been studied particularly well. Both can function very early in the pathway of spliceosome assembly (Fu and Maniatis 1990; Krainer et al. 1990b), and evidence has been presented that each is able by itself to commit a pre-mRNA to the splicing pathway (Fu 1993). This may reflect the ability of purified ASF/SF2 to recognize 5' splice sites (Zuo and Manley 1994) and to bind 5' splice sites cooperatively with Ul snRNP (Eperon et al. 1993; Kohtz et al. 1994; Jamison et al. 1995; Zahler and Roth 1995). This cooperative binding likely reflects a proteinprotein interaction between ASF/SF2 and a component of the snRNP, the U1 70K protein (Kohtz et al. 1994). It has also been shown (Wu and Maniatis 1993) that both ASF/SF2 and SC35 are able to interact simultaneously with $70 \mathrm{~K}$ and $\mathrm{U} 2 \mathrm{AF}$, a factor that facilitates U2 snRNP binding to the pre-mRNA branch site (Zamore and Green 1989). This supports the view that another function of SR proteins may be to help bring together $5^{\prime}$ and $3^{\prime}$ splice sites (Fu and Maniatis 1992b). SR proteins assembled on a splicing enhancer can also facilitate U2AF binding to the pre-mRNA (Wang et al. 1995; Zuo and Maniatis 1996). Finally, addition of excess SR proteins to nuclear extracts can overcome the requirement of U1 snRNP (Crispino et al. 1994; Tarn and Steitz 1994), raising the possibility that the U1 snRNP-ASF/SF2 cooperative binding may function as much to recruit SR proteins to the $5^{\prime}$ splice site as the other way around. SR proteins bound at the $5^{\prime}$ splice site then likely function at subsequent stages in assembly of the spliceosome (Roscigno and Garcia-Blanco 1995; Tarn and Steitz 1995).

Whereas the functions of SR proteins have been well studied in vitro, it has been more difficult to demonstrate the role of these proteins in vivo. Consistent with a function in splicing, SR proteins colocalize in the nucleus with known splicing factors (reviewed by Spector 1993). Experiments utilizing transient cotransfection assays have shown that overexpressed SR proteins can influence the accumulation of mRNAs produced from alternatively spliced reporter transcripts in a fashion largely, but not entirely, consistent with expectations from in vitro experiments (Cáceres et al. 1994; Wang and Manley 1995; Screaton et al. 1995). In the absence of yeast as a model system, genetic analysis of SR proteins has to date been limited to one gene in Drosophila, B52. $B 52$ null alleles display a lethal phenotype during development, indicating that $B 52$ provides at least one nonredundant function necessary for proper development (Ring and Lis 1994; Peng and Mount 1995). However, no defects in gene expression were detected in homozygous mutant larvae, leaving unclear the basis for the developmental failure.
Here we describe development of a genetic system to study the function of the SR protein ASF/SF2 in vivo. For this, we employed the chicken B-cell line DT40. These cells have been shown previously to undergo homologous recombination at an exceptionally high frequency (Buerstedde and Takeda 1991). This property, coupled with their rapid generation time (8-9 hr), makes them ideal for certain genetic experiments, and they have in fact been used previously for disruption and characterization of a number of nonessential genes (e.g., Buerstedde and Takeda 1991; Takeda et al. 1992; Takata et al. 1994; Li and Dodgson 1995). Here we exploit the properties of DT40 cells to show that the gene encoding ASF/ SF2 is essential for cell viability. We describe conditions that allow targeted disruption of both alleles of $A S F / S F 2$, in the presence of a single copy of an ASF/SF2 cDNA under the control of a repressible promoter. Inactivation of the promoter caused depletion of ASF/SF2, accumulation of incompletely processed pre-mRNA, cessation of growth, and subsequent cell death. We also describe a method that allows analysis of the ASF/SF2 domains required to support cell growth, and present evidence that ASF/SF2 mRNA levels are tightly autoregulated. The system we describe should prove useful for study of other essential vertebrate genes.

\section{Results}

To begin a genetic analysis of $A S F / S F 2$, we first cloned chicken $A S F / S F 2$ ( $C A S F / S F 2)$ cDNAs and the genomic DNA. Human and chicken ASF/SF2 are $98 \%$ identical at the amino acid level and $78 \%$ identical at the nucleotide level. Like the mouse gene (J. Kohtz and J.L. Manley, unpubl.), the $c A S F / S F 2$ gene contains four introns, one of which lies in the $3^{\prime}$ untranslated region (UTR; Fig. 1C). As described previously for human $A S F / S F 2$ cDNAs (Ge et al. 1991), cDNAs retaining the third intron, capable of encoding a truncated protein, were isolated. Expression of ASF/SF2 in DT40 cells was confirmed by Northern blot and Western blot analyses. $C A S F / S F 2$ is a single-copy gene as determined by Southern blot analysis (data not shown).

\section{ASF/SF2 is an essential gene in DT40 cells}

To begin targeted disruption of $c A S F / S F 2$, bacterial hygromycin and neomycin-resistance genes, each driven by the chicken $\beta$-actin promotor, were inserted into the first exon of $c A S F / S F 2$ genomic DNA, giving the constructs Hygro-ASF/SF2 and Neo-ASF/SF2, respectively (Fig. 1C). DT40 cells were transfected with Hygro-ASF/ SF2 and 30 hygromycin-resistant clones were isolated and screened for homologous recombination by Southern blotting. Hybridization with probe 1 (Fig. 1C) showed that EcoRI-digested DNA from 25 of the 30 clones gave rise to a new $4.5-\mathrm{kb}$ band, the size expected following homologous recombination (Fig. $1 \mathrm{~A}$ and data not shown). DNAs from these 25 clones were then digested with KpnI and hybridized with probe 2 (Fig. 1C). Providing additional evidence for homologous recombination, a 
A

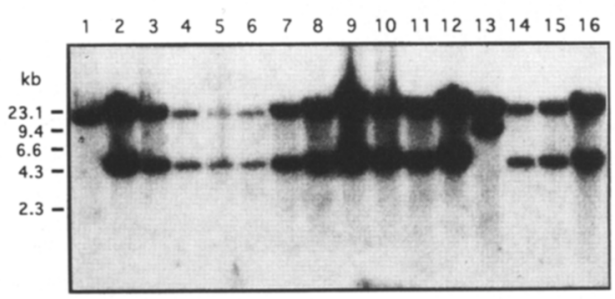

B

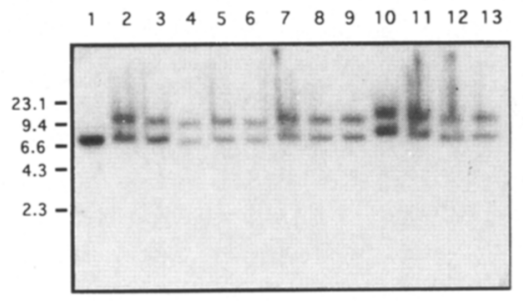

C

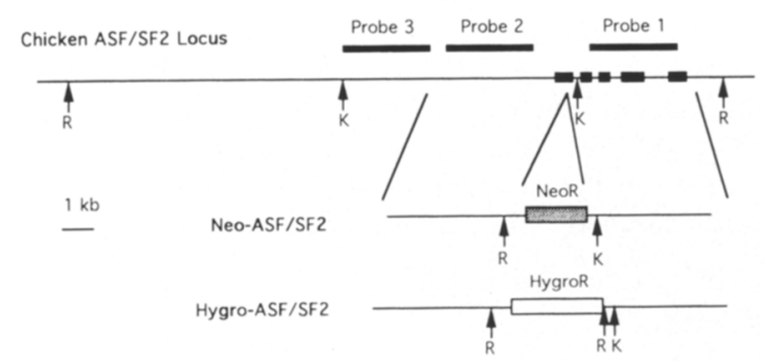

Figure 1. High-frequency disruption of a single $A S F / S F 2$ allele. (A) Wild-type DT40 cells were transfected with the construct Hygro-ASF/SF2. Genomic DNAs from untransfected DT40 cells (lane 1) and 15 hygromycin-resistant clones (lanes 2-16) were digested with EcoRI and hybridized with probe 1. All clones but one (lane 13 ) gave rise to a 4.5 - $\mathrm{kb}$ band consistent with homologous combination. $(B)$ Genomic DNAs from untransfected DT40 cells (lane 1) and 12 of the clones (lanes 2-13) that showed a $4.5-\mathrm{kb}$ band in $A$ were digested with $K p n I$ and hybridized with probe 2 . The positions of lambda DNA/HindIII markers are indicated. $(C)$ Restriction map of the chicken ASF/ SF2 locus and constructs used for targeted disruption. Shaded boxes represent exons of the chicken ASF/SF2 gene. Relevant restriction enzyme sites are shown: (R) EcoRI; (K) KpnI. The positions of the probes used in Southern blot analysis are indicated. (NeoR) Neomycin-resistance gene; (HygroR) Hygromycin-resistance gene.

new band of $10 \mathrm{~kb}$ appeared in all 25 clones (Fig. 1B and data not shown). Probe 3 (Fig. 1C), which is complementary to the flanking region of the insertion site, also detected a 10-kb fragment after KpnI digestion (data not shown). These results indicate that targeted disruption of one allele of the $A S F / S F 2$ gene occurred at a frequency of $\sim 80 \%$.

If ASF/SF2 is not essential for cell viability, a second round of gene targeting should disrupt the second $A S F /$ $S F 2$ allele. To test this, one of the 25 heterozygous clones was transfected with Neo-ASF/SF2 and selected in medium containing both hygromycin and G418. DNAs from 37 resistant clones were analyzed by Southern blot.
The results showed that none of them underwent homologous recombination (Table 1 and data not shown). To confirm that Neo-ASF/SF2 was capable of homologous recombination, we transfected wild-type DT40 cells with this construct. Out of 39 G418-resistant clones, 16 had targeted disruption of one ASF/SF2 allele (Table 1 and data not shown), giving a frequency of $\sim 40 \%$. To provide further evidence that the second $A S F /$ SF2 allele could not be disrupted, heterozygous DT40 cells with one allele disrupted by Neo-ASF/SF2 were transfected with Hygro-ASF/SF2. None of 42 resistant clones underwent homologous recombination (Table 1 and data not shown). Taken together, these data suggest that $A S F / S F 2$ is an essential gene in DT40 cells.

\section{Human ASF/SF2 can functionally substitute for its chicken homolog}

If our inability to disrupt the second $A S F / S F 2$ allele was because ASF/SF2 is essential for cell viability, then it should be possible to target the gene when the protein is supplied exogenously. To test this, mutant DT40 cells with one $A S F / S F 2$ allele disrupted (by Neo-ASF/SF2) were stably transfected with a vector expressing human ASF/SF2 (hASF/SF2) from an ASF/SF2 cDNA, and also providing puromycin resistance (Puro-ASF; see Materials and Methods). hASF/SF2 was tagged with an influenza HA epitope (flu) at the amino terminus to facilitate its detection. Southern blots indicated random integration of the transgene at sites not linked to the $C A S F / S F 2$ locus (results not shown). Western blot analysis using an antiflu monoclonal antibody (Field et al. 1988) showed that flu-hASF/SF2 was expressed in many clones, although at somewhat different levels (Fig. 2A). Cells from a clone giving high expression of flu-hASF/SF2 (equivalent to lane 10 of Fig. 2) were transfected with Hygro-ASF/SF2 and selected in medium containing hygromycin and G418. DNAs from 43 doubly resistant clones were analyzed by Southern blotting after EcoRI digestion using probe 1. The results (Fig. 2B and data not shown) indicated that 17 clones contained a $4.5-\mathrm{kb}$ fragment, the expected size after homologous integration, and had lost the wild-type $20-\mathrm{kb}$ fragment. Southern blot analysis after KpnI digestion using probe 3 confirmed that these 17 clones underwent homologous recombination (data not

Table 1. Frequencies of homologous recombination in Hygro-ASF/SF2 and Neo-ASF/SF2 transfected DT4O cells

\begin{tabular}{lcc}
\hline Transfections & Hygro-ASF/SF2 & Neo-ASF/SF2 \\
\hline First round & $25 / 30$ & $16 / 39$ \\
Second round & $0 / 42^{\mathrm{a}}$ & $0 / 37^{\mathrm{b}}$
\end{tabular}

DT40 cells were transfected with the indicated plasmids and drug-resistant clones were analyzed for homologous integration by Southern blotting (see Materials and Methods).

${ }^{a}$ Heterozygous DT40 cells with one ASF/SF2 allele disrupted with Neo-ASF/SF2 were transfected.

${ }^{b}$ Heterozygous DT40 cells with one ASF/SF2 allele disrupted with Hygro-ASF/SF2 were transfected. 
A

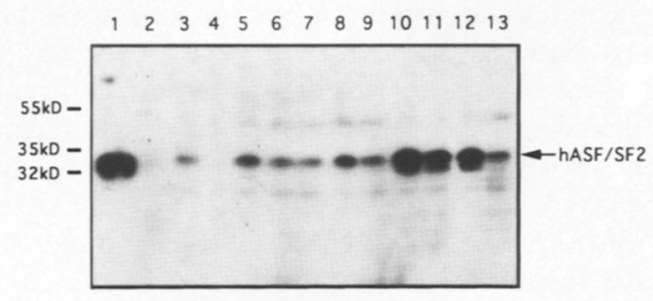

B

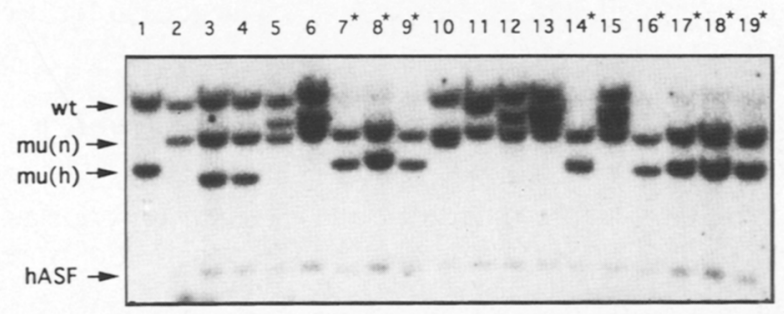

Figure 2. Complete disruption of chicken $A S F / S F 2$ in the presence of a human ASF/SF2 cDNA. (A) Expression of human ASF/ SF2 in heterozygous DT40 cells. A mutant DT40 cell line, in which one ASF/SF2 allele had been disrupted by NeoR, was transfected with Puro-ASF (see Materials and Methods). Lysates from human 293 cells (lane 1) transfected with a plasmid encoding flu-hASF/SF2, pCF21 (Wang and Manley 1995), and 12 puromycin-resistant DT40 clones (lanes 2-13) were analyzed for protein expression by antiflu monoclonal antibody, $12 \mathrm{CA} 5$. The positions of protein markers and flu-hASF/SF2 are indicated. $(B)$ Southern blot analysis of targeted integration. A DT40 cell line in which one $A S F / S F 2$ allele had been disrupted by NeoR, and which contains and expresses flu-hASF/SF2 cDNA, was transfected with the construct Hygro-ASF/SF2. Genomic DNAs from doubly resistant clones (lanes 3-19) were digested with $E c o$ RI and hybridized with probe 1. Genomic DNAs from DT40 cells in which one $A S F / S F 2$ allele had been disrupted by HygroR (lane 1) or NeoR (lane 2), were used as controls. Positions of fragments from wild-type (wt), NeoR-disrupted [mu(n)] and HygroR-disrupted $[\mathrm{mu}(\mathrm{h})] A S F / S F 2$ alleles are indicated. Human ASF/SF2 cDNA (hASF) was also detected. Asterisks indicate complete knockouts.

shown). We randomly chose one clone and analyzed the level of cASF/SF2 mRNA by RNase protection of total cellular RNA, using a probe complementary to the first exon and intron of CASF/SF2 gene (Fig. 3, bottom; the probe does not protect hASF/SF2 mRNA). No cASF/SF2 mRNA could be detected in the double knockout clone [Fig. 3A, lane 4; in this and all RNase protecion analyses shown, the integrity of the RNAs was verified by visualization of the 28 and $18 \mathrm{~S}$ rRNA species (data not shown)].

Taken together, our data indicate that both alleles of chicken $A S F / S F 2$ can be disrupted in the presence, but not the absence, of an $h A S F / S F 2$ transgene. These results confirm that ASF/SF2 is required for DT40 cell viability, and indicate that hASF/SF2 can substitute functionally for its chicken homolog. In addition, forms of ASF/SF2

that arise by alternative splicing, ASF-2 and ASF-3 /Ge et al. 1991), cannot be produced from the transgene, and thus are not essential for viability.

The level of ASF/SF2 mRNA is controlled by an autoregulatory loop

The RNase protection data in Figure 3A provided additional insights into ASF/SF2 expression. First, wild-type DT40 cells and the heterozygous cells containing only a single $A S F / S F 2$ allele produced equivalent amounts of ASF/SF2 mRNA (cf. lanes 1 and 2 and data not shown), suggesting the existence of a regulatory mechanism capable of maintaining ASF/SF2 transcripts at a constant

A

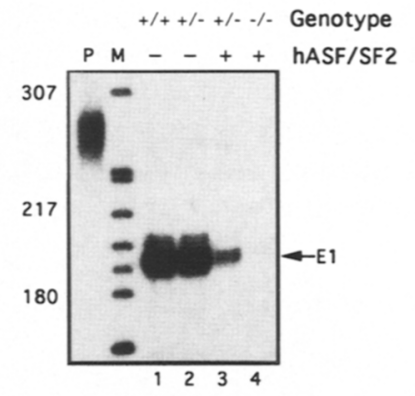

B
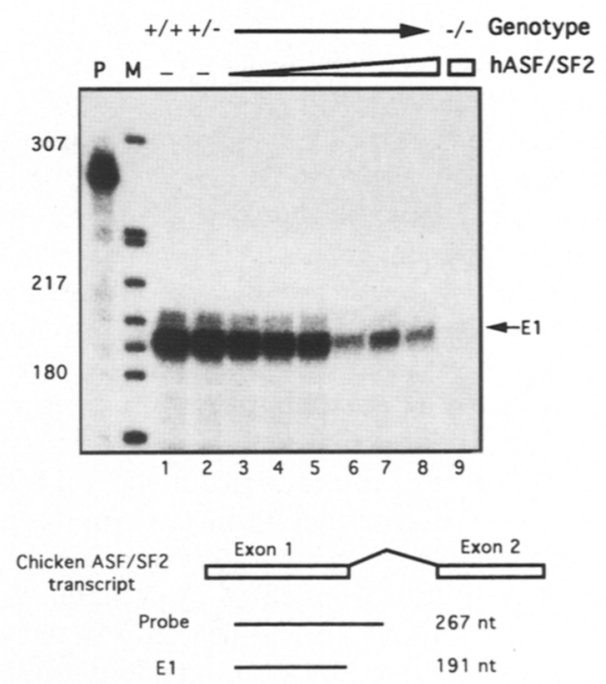

Figure 3. RNase protection analysis of chicken ASF/SF2 mRNA in different DT40 clones. $(A)$ Total RNAs from wildtype DT40 cells (lane 1), a heterozygous line (lane 2), the heterozygote expressing flu-hASF/SF2 (lane 3), and double knockout cells (lane 4) were analyzed by RNase protection, as diagrammed at the bottom. Numbers indicate the nucleotide length of the probe and the protected fragments. $(B)$ Total RNAs from wild-type DT40 cells (lane 1), a heterozygous line (lane 2), subclones of the heterozygote expressing different levels of fluhASF/SF2 (lanes 3-8), and double knockout cells (lane 9) were analyzed as in $A$. The genotype of $A S F / S F 2$ and the approximate levels of exogenously expressed hASF/SF2 protein are indicated at top. (P) Probe; (M) ${ }^{32}$ P-labeled pBR322/HpaII marker. Numbers besides the figures indicate size of the DNA marker. 
level. Providing additional evidence for this idea, the heterozygous cells expressing ASF/SF2 from the human transgene accumulated significantly less endogenous cASF/SF2 mRNA than did the parental heterozygous cells lacking the transgene (cf. lanes 2 and 3). To test whether this apparent down-regulation of endogenous cASF/SF2 mRNA is a general property of clones expressing the transgene, and not a peculiarity of the line analyzed in Fig. 3A, we measured accumulation of cASF/ SF2 mRNA in a number of the heterozygous, transgenic lines analyzed for hASF/SF2 protein levels in Figure $2 \mathrm{~A}$. The results of RNase protection experiments (Fig. $3 \mathrm{~B}$ ) indicate that the accumulation of cASF/SF2 mRNA was reduced significantly in the three cell lines that expressed high levels of hASF/SF2 protein (lanes 6-8), but not in the clones expressing lower amounts of hASF/SF2 (lanes 3-5). The reduction in cASF/SF2 mRNA levels did not reflect a nonspecific inhibitory effect resulting from high levels of ASF/SF2, as accumulation of two other endogenous transcripts was not affected (data not shown). These results suggest that CASF/SF2 mRNA levels are controlled by an autoregulatory loop designed to maintain constant amounts of the protein.

\section{Creation of a conditional ASF/SF2 allele}

The experiments described above provide a means for disrupting both alleles of an essential gene in DT40 cells, by introducing a functional transgene prior to disruption of the second allele. This allowed us to show that $A S F /$ $S F 2$ is in fact an essential gene, and to examine the consequences of varying gene dosage. However, considerably more information could be obtained if expression of the transgene was conditional instead of constitutive. To this end, we adapted the tetracycline (tet)-repressible promoter system, described by Gossen and Bujard (1992), to DT40 cells. Briefly, this system requires two transgenes, one constitutively expressing a chimeric activator consisting of the bacterial tet repressor DNA binding domain fused to the viral VP16 transcriptional activation domain (tetR-VP16), and the other capable of expressing flu-ASF/SF2 from a minimal promoter consisting essentially of a TATA box and binding sites for the tetR-VP16 activator (the tet operator, tetO). The minimal promoter is inactive in the absence of functional tetR-VP16, which itself is inactive in the presence of tet, as tet will bind tetR and prevent DNA binding. Previous work with this system has been with mammalian and plant cells, and we were therefore confident it would function in a similar manner in chicken cells.

To establish tet-repressible synthesis of ASF/SF2 in DT40 cells, we first constructed vectors in which the tetR-VP16 and flu-hASF/SF2 cDNAs were under control of the chicken $\beta$-actin promoter and the tetO-minimal promoter, respectively, giving vectors tTA and $\mathrm{mASF} / \mathrm{SF} 2$. tTA also contains the puromycin-resistance gene, and $\mathrm{mASF} / \mathrm{SF} 2$ expresses histidinol-resistance. Heterozygous DT40 cells with one $A S F / S F 2$ allele disrupted by Neo- ASF/SF2 were cotransfected with tTA and $\mathrm{mASF} / \mathrm{SF} 2$ and selected in medium containing puro- mycin and histidinol. Six doubly resistant clones were isolated and analyzed. To test for tet-repressible synthesis of flu-hASF/SF2, the cells were grown for $48 \mathrm{hr}$ in the presence or absence of tet, and whole-cell lysates were prepared and screened by Western blotting using the antiflu antibody as described above. Strikingly, five clones displayed efficient tet-repressible expression of fluhASF/SF2, with the protein reduced to undetectable levels after $48 \mathrm{hr}$ (Fig. 4A and results not shown). The second $A S F / S F 2$ allele was then disrupted in one of these clones, using Hygro-ASF/SF2 as described above (see Fig. 4B). Several cell lines in which the only copy of $A S F / S F 2$ is expressed from a tet-repressible promoter were obtained, and one, named \#3, was chosen for further analysis.

\section{Depletion of ASF/SF2 results in arrest of cell growth and cell death}

We first wished to determine the effect of tet on accumulation of hASF/SF2 in $\# 3$ cells as a function of time.

A

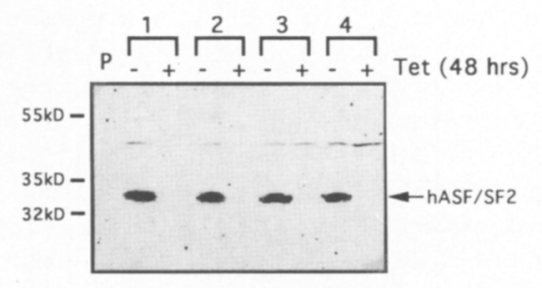

B

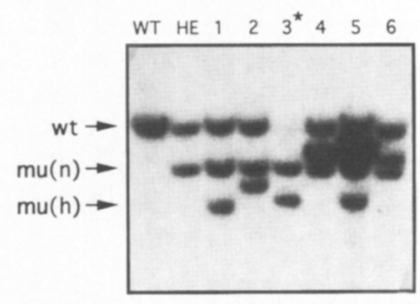

Figure 4. Expression of human ASF/SF2 from a tetracyclinerepressible promoter. $|A|$ Heterozygous DT40 cells in which one $A S F / S F 2$ allele had been disrupted by NeoR were cotransfected with vectors $\mathrm{tTA}$ and $\mathrm{mASF} / \mathrm{SF} 2$ and selected with puromycin and histidinol. Four doubly resistant clones were grown in the absence or presence of tet $(1 \mu \mathrm{g} / \mathrm{ml})$ for $48 \mathrm{hr}$. Lysates from these clones were analyzed by Western blotting as in Fig. 2A. Lysate prepared from the parental heterozygous cells $(\mathrm{P})$ was used as negative control. Positions of protein markers and hASF/SF2 are indicated. $|B|$ One of the four clones analyzed in $A$ was transfected with the construct Hygro-ASF/SF2 and selected with G418 and hygromycin. DNAs from six doubly resistant clones were analyzed by Southern blotting as in Fig. 2B. Wildtype cells (WT) and heterozygotes with one $A S F / S F 2$ allele disrupted by NeoR (HE) were used as controls. Positions of fragments from wild-type $(\mathrm{wt})$, NeoR-disrupted [mu(n)] and HygroR-disrupted $[\mathrm{mu}(\mathrm{h})] A S F / S F 2$ allele are indicated. Asterisk indicates a complete knockout, named \#3. 
A

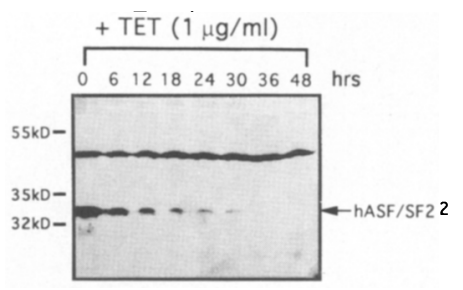

B

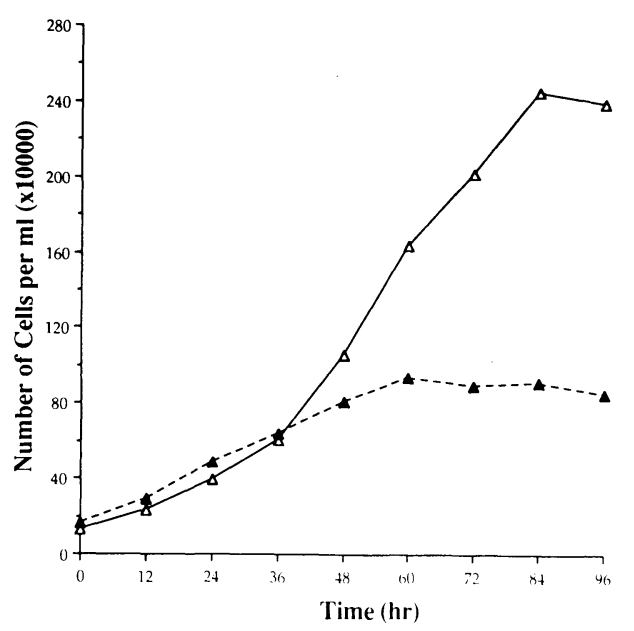

Figure 5. Depletion of ASF/SF2 results in arrest of cell growth. (A) \#3 cells were transferred into medium containing $1 \mu \mathrm{g} / \mathrm{ml}$ tet. Lysates prepared after incubation of $0,6,12,18,24,30,36$, and $48 \mathrm{hr}$ were analyzed for hASF/SF2 expression by Western blotting as in Fig. 2A. The time points are indicated at top. Positions of protein markers and hASF/SF2 are indicated. $(B)$ Growth curve of \#3 cells in presence or absence of tet. \#3 cells were transferred to medium with or without $1 \mu \mathrm{g} / \mathrm{ml}$ tet, and the concentrations of cells at different time points were determined by counting with a hemacytometer. $(\Delta)$ Cell growth in the absence of tet; $(\mathbf{\Delta})$ growth in the presence of tet.

Cells were grown in medium containing $1.0 \mu \mathrm{g} / \mathrm{ml}$ tet and analyzed for flu-hASF/SF2 by Western blotting at different time points following addition of tet. As shown in Fig. 5A, the amount of hASF/SF2 decreased gradually between 0 and $30 \mathrm{hr}$, and could not be detected after $36 \mathrm{hr}$.

We next examined cell growth and viability following addition of tet to the medium. \#3 cells were split to $\sim 1.5 \times 10^{5} / \mathrm{ml}$, and then grown in the presence or absence of $1.0 \mu \mathrm{g} / \mathrm{ml}$ tet. In the absence of tet, $\# 3$ cells grew until reaching confluency, which is $\sim 2.5 \times 10^{6}$ cells $/ \mathrm{ml}$ (Fig. 5B). In contrast, in the presence of tet, the cells ceased growing between 48 and $60 \mathrm{hr}$, at a density of $\sim 8 \times 10^{5} / \mathrm{ml}$ (Fig. 5B). Based on trypan blue exclusion (data not shown), the cells began to die between 60 and $72 \mathrm{hr}$ after addition of tet, and were nearly all dead by 96 hr. Tet had no effect on the growth or viability of either wild-type DT40 cells or heterozygous cells expressing tetR-VP16 and flu-hASF/SF2 (results not shown). We conclude from this data that the presence of tet results in depletion of ASF/SF2, which in turn results in growth arrest and subsequent cell death.

\section{Depletion of ASF/SF2 results in a pre-mRNA processing defect}

We next wished to determine whether depletion of ASF/ SF2 leads to a detectable pre-mRNA splicing defect. We first examined several specific transcripts, isolated from \#3 cells grown in tet for different times, by nuclease S1 mapping and/or Northern analysis. The results in each case revealed a disappearance of mRNA, beginning at 24 $\mathrm{hr}$ following the addition of tet, but accumulation of unspliced pre-mRNA could not be detected (results not shown). Although this likely reflects instability of the unprocessed pre-mRNA, we wished to obtain more direct evidence for a splicing defect, and therefore took a different approach. We reasoned that, if unprocessed premRNA is in fact rapidly degraded, we might be able to detect unspliced RNA if we directly examined pulse-labeled RNA, as opposed to analyzing accumulated, steady-state RNA. We therefore performed preliminary experiments to optimize labeling times and other parameters. Based on these results, \#3 cells were grown in the absence of tet for $24 \mathrm{hr}$ or in the presence of tet for 24 or $48 \mathrm{hr}$, and then labeled with ${ }^{3} \mathrm{H}$ uridine for $30 \mathrm{~min}$, as described in Materials and Methods. Total cell RNAs were isolated, subjected to two rounds of selection on oligo(dT) cellulose, and resolved by denaturing agarose gel electrophoresis. Densitometric scans of the resulting fluorograms are shown in Figure 6. Strikingly, after $24 \mathrm{hr}$ growth in tet (which is sufficient to deplete $80-90 \%$ of the ASF/SF2; see Fig. 5A), there was a substantial accumulation of extremely large (significantly lower mobility than the $45 \mathrm{~S}$ pre-rRNA marker) poly(A) ${ }^{+}$RNA that was not detected in the RNA sample from the cells grown in the absence of tet. The amount of this high

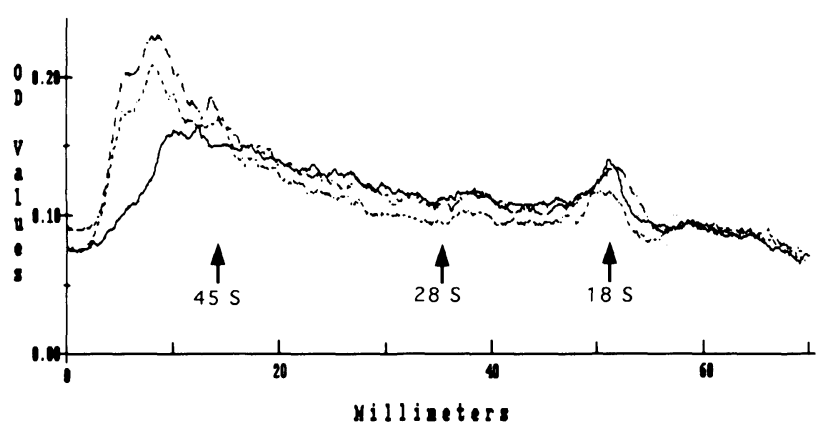

Figure 6. Depletion of ASF/SF2 results in a pre-mRNA processing defect. \#3 cells grown either in the absence of tet (solid line) or in the presence of tet for 24 (dotted line) or $48 \mathrm{hr}$ (dashed line) were labeled with ${ }^{3} \mathrm{H}$-uridine for $30 \mathrm{~min}$. Poly $(\mathrm{A})^{+} \mathrm{RNAs}$ were isolated and resolved on a formaldehyde agarose gel (see Materials and Methods), and the resulting fluorograph was scanned with a densitometer. Distances migrated (in $\mathrm{mm}$ ) are indicated on the horizontal axis. Positions of $45 \mathrm{~S}$ pre-rRNA, and $28 \mathrm{~S}$ and $18 \mathrm{~S}$ rRNAs are indicated. 
molecular weight RNA increased in the cells labeled after 48-hr growth in the presence of tet. Similar results have been observed in multiple, independent experiments. Given that there were no reductions in the recovery of poly $(\mathrm{A})^{+}$RNA (i.e., there was no detectable effect on polyadenylation), and no effects on incorporation of ${ }^{3} \mathrm{H}$ uridine into RNA (i.e., transcription was unaffected), our results suggest strongly that depletion of ASF/SF2 interferes with pre-mRNA splicing. Furthermore, given that the defect was readily detectable with total poly $(\mathrm{A})^{+}$RNA, we conclude that ASF/SF2 is essential for efficient processing of a significant fraction of the cell's transcripts.

Rescue of tet-induced lethality provides an assay for ASF/SF2 function

The tet-induced lethality displayed by \#3 cells suggests the possibility of establishing a genetic complementation assay based on rescue of the lethal phenotype. We reasoned that transformation of \#3 cells with plasmids capable of expressing proteins that can provide the ASF/ SF2 essential function should allow growth (i.e., formation of colonies) in the presence of tet. The feasibility of this approach was verified using a plasmid expressing hASF/SF2 under control of the chicken $\beta$-actin promoter. Transformation of \#3 cells with this plasmid followed by selection in the presence of tet gave rise to 32 colonies /this is similar to the numbers obtained with other drugs, as described above), whereas no colonies were obtained from mock transformed cells or cells transformed with the vector alone (Fig. 7 and results not shown).

We next determined the ability of a series of expression plasmids to rescue tet-induced lethality. Plasmids encoding either other human SR proteins, SC35 and SRp40, or six different mutant derivatives of ASF/SF2, which had been studied previously using in vitro assays (Zuo and Manley 1993; see Fig. 7), were constructed and used to transfect \#3 cells. Neither SC35 nor SRp40 gave rise to any tet-resistant colonies (Fig. 7). This strengthens the conclusion that ASF/SF2 function is not redundant, as ASF/SF2 is essential even under conditions that should allow overexpression of another SR protein. Amongst the mutants, only the derivative called Gly, which lacks a glycine-rich region separating the two RBDs, was functional, and it was as effective as wildtype in rescuing tet-induced lethality. This is consistent with its behavior in vitro, where it functioned as well as wild-type ASF/SF2 in S100 complementation and splicesite switching assays (Zuo and Manley 1993). The other, inactive mutants were all defective in vitro (at least in the S100 assayl, with one exception. The mutant RS-b, which lacks the carboxy-terminal half of the RS domain, was observed previously to be fully active in vitro. The discrepancy is unlikely to reflect either protein instability, as accumulation of RS-b (as well as the other mutants tested) and hASF/SF2 was similar when measured in stably transformed DT40 cells (results not shown), or defective subcellular localization, as the proteins dis-

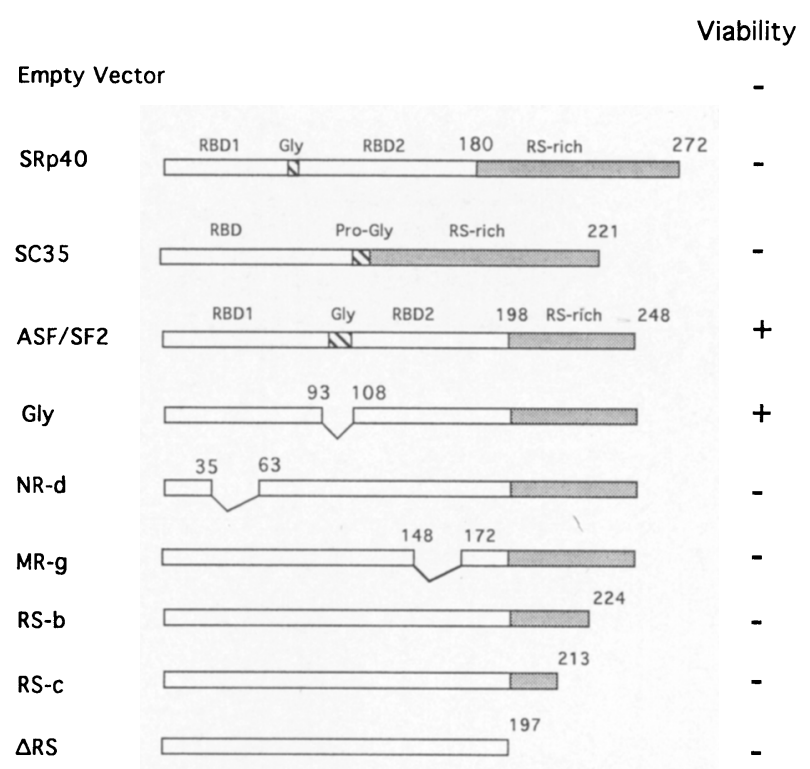

Figure 7. Complementation of tet-induced lethality by SR proteins and ASF/SF2 mutant derivatives. \#3 cells were transfected with empty vector (PA-Puro) or expression vectors encoding SRp40, SC35, ASF/SF2, and ASF/SF2 mutant derivatives, and then selected in medium containing $1 \mu \mathrm{g} / \mathrm{ml}$ tet. $(-)$ No surviving clones; $(+)$ appearance of surviving colonies; (RBD) RNP-type RNA binding domain; (RS-rich) arginine/ serine-rich region; (Pro-Gly) proline/glycine-rich region; (Gly) glycine-rich region. Numbers indicate amino acid residues. Positions of deleted residues are shown.

played similar nuclear localization patterns when overexpressed by transient transfection in human 293 cells (Wang and Manley 1995). Our results thus suggest that the entire ASF/SF2 RS domain plays an important role in splicing, but in any event indicate that it is essential for cell viability.

\section{Discussion}

We have taken advantage of the properties of chicken DT40 cells to show that the gene encoding the SR protein ASF/SF2 is essential for viability. Our strategy was to perform a series of transformations that resulted in disruption of both alleles of the gene, which was made possible by the prior insertion of a transgene expressing a conditional (tet repressible) ASF/SF2 cDNA. To our knowledge, this represents the first targeted disruption of an essential gene in cultured vertebrate cells. Our results have both provided insights into the properties of ASF/SF2 in vivo, and also established a system that should be of value for the genetic analysis of other essential vertebrate genes.

Our finding that ASF/SF2 is essential for cell viability is consistent with some previous studies, but might not have been predicted by others. As described in the introduction, early in vitro studies indicated that ASF/SF2 function could be provided by other SR proteins in several different assays. However, other assays using more 
sensitive assays such as splicing commitment (Fu 1993) or enhancer-dependent splicing (e.g., Sun et al. 1993) have suggested that ASF/SF2 may provide a unique function during splicing of certain pre-mRNAs. We mentioned above that the Drosophila SR protein B52 is essential for proper development, with lethality observed in the late embryo/early larval stage (Ring and Lis 1994; Peng and Mount 1995). Although this is consistent with B52 providing a function required for cell viability, it is also possible that lethality resulted from a defect in a specific process required for proper development but not for cell growth. It is conceivable that this might be the case with another RNA binding protein suggested to play a role in pre-mRNA splicing, the hnRNP Al protein (Al). Al was shown to function in certain alternative splice site selection assays in a manner competitive with ASF/ SF2 (Mayeda and Krainer 1992; Cáceres et al. 1994; Yang et al. 1994). However, unlike ASF/SF2 in DT40 cells, A1 expression was found to be dispensable for normal growth of a cultured mouse erythroleukemia cell line (Ben-David et al. 1992). In contrast, a possible Al homolog in Drosophila, squid/hrp40, is, like B52, essential for proper development (Matunis et al. 1992; Kelley 1993). Although it may be that squid/hrp40 is not in fact the true homolog of A1 (e.g., Haynes et al. 1990), it is also possible that the protein has an essential function in development but not in cell growth.

Our data also indicate that the levels of ASF/SF2 mRNA (and therefore presumably protein) are tightly controlled. That wild-type and heterozygous cells displayed the same levels of ASF/SF2 mRNA could indicate that mRNA production from the wild-type allele is elevated by an autoregulatory mechanism, to maintain constant levels of ASF/SF2 protein. More indicative of autoregulation, though, is the observed repression of endogenous ASF/SF2 mRNA in the presence of elevated levels of ASF/SF2 protein expressed from the exogenous transgene. Although we have not been able to detect accumulation of unspliced ASF/SF2 pre-mRNA, we nonetheless believe that regulation occurs at the level of splicing. In Drosophila, known genes encoding splicing regulators are all thought to be autoregulated at the level of splicing (reviewed by Mattox et al. 1992), so it would in fact be surprising if the ASF/SF2 autoregulation involved a nonsplicing mechanism. There are several examples where ASF/SF2 has been suggested to function negatively. We showed previously that transient overexpression of ASF/SF2 significantly inhibited accumulation of SV40 early mRNA synthesized from a cotransfected reporter plasmid, likely reflecting inhibition of splicing (Wang and Manley 1995). It has also been reported that ASF/SF2 binds to a negative regulator of splicing in Rous Sarcoma virus RNA (McNally and McNally 1996), and can inhibit in vitro splicing of an adenovirus pre-mRNA by binding to an intronic repressor element (Kanopka et al. 1996). Thus negative regulation of splicing may in fact be a natural function of ASF/SF2, which could be employed in autoregulation. That overexpression of an SR protein can be deleterious has been demonstrated in Drosophila, where forced overexpres- sion of B52 in a number of different tissues was shown in many cases to cause serious developmental defects (Kraus and Lis 1994).

In addition to establishing that ASF/SF2 is essential for cell viability, our data has provided evidence that a biochemically defined metazoan splicing factor is in fact required for pre-mRNA processing in vivo. The processing defect we detected is likely to reflect a direct effect on splicing. At $24 \mathrm{hr}$ after tet addition, when accumulation of high molecular RNA was readily apparent, defects in cell growth or other measurable parameters have not been detected. For example, neither transcription nor $3^{\prime}$ end formation is affected at this time, based on incorporation of ${ }^{3} \mathrm{H}$ uridine into total and poly $(\mathrm{A})^{+}$RNA during short (15-60 min) pulse-labeling periods (unpubl.). Ring and Lis (1994) were unable to detect an RNA processing defect in $B 52$ homozygous null mutant larvae, by Northern blot analysis of five specific transcripts. This could indicate that B52 is not essential for splicing, at least for the RNAs tested. Alternatively, technical difficulties inherent in the system (e.g., the persistence of maternal B52 and the necessity of analyzing steady-state RNA) may have prevented detection of a splicing defect. Supporting the view that B52 does function in splicing in vivo, Peng and Mount (1995) presented evidence that a dominant gain-of-function $B 52$ allele can both enhance the phenotypes of several mutations thought to affect splicing, as well as alter the pattern of sex-specific splicing of doublesex pre-mRNA.

Our results have provided insights into the isoforms and domains of ASF/SF2 required for cell viability. Given that cells in which the sole source of ASF/SF2 is a cDNA grow normally, the ASF-2 and ASF-3 isoforms, which would arise by alternative splicing $/ \mathrm{Ge}$ et al. 1991), are not required for cell viability. ASF-2 would result from use of an alternative $3^{\prime}$ splice site in the third intron, and would contain a distinct non-RS carboxyterminal domain by translation of the fourth exon in an alternative reading frame. Intriguingly, the putative alternative $3^{\prime}$ splice and open alternative reading frame are conserved in chicken, mouse, and humans, implying some function. The ability to synthesize ASF-3, which is a truncated form of the protein lacking the RS domain that would be produced by retention of the third intron, is likewise conserved amongst these three species, and appropriate cDNAs have been isolated. It is possible that these isoforms perform a function in DT40 cells not required for viability, or are only expressed or necessary in other specific tissues or developmental stages. With respect to domains of the protein, only the glycine-rich hinge domain was found to be dispensable. This was on the one hand surprising because, similar to the case with ASF-2 and ASF-3, this sequence is highly conserved during evolution. On the other hand, this same mutation did not have a detectable effect on the protein's activities in vitro (Zuo and Manley 1993). Perhaps the flexibility presumed to be imparted by this domain is important only under growth conditions not reflected in our assays.

Our results indicating an important role for the ASF/ SF2 RS domain are significant for several reasons. First, 
in vitro experiments showed that the RS domain for at least part of it) is essential for ASF/SF2 to function as an essential splicing factor in the $\mathrm{S} 100$ complementation assay, but is dispensable in splice site switching assays (Cáceres and Krainer 1993; Zuo and Manley 1993). The RS domain could also be deleted without significant effect in a cotransfection assay monitoring splice site switching of an adenovirus pre-mRNA /Wang and Manley 1995). Although it remains possible that the RS domain is in fact unnecessary for certain functions performed by ASF/SF2, the results of our in vivo complementation assays highlight its importance for at least one function required for cell viability. Indeed, the requirement for the RS domain appears even more stringent than suggested by the in vitro results, as judged by the behavior of the RS-b mutant. As RS-b has not shown a defect in any in vitro assays, including as a target for in vitro phosphorylation (Woppmann et al. 1993) and in cooperating with U1 snRNP in $5^{\prime}$ splice site recognition (Jamison et al. 1995), in addition to S100 complementation (Zuo and Manley 1993), the function of the 24 carboxy-terminal residues deleted in RS-b thus remains to be elucidated.

Regions with similarity to RS domains are found in a number of splicing factors in addition to SR proteins /reviewed by Fu 1995). One such region is in the large subunit of $\mathrm{U} 2 \mathrm{AF}\left(\mathrm{U} 2 \mathrm{AF}^{65}\right)$. The region is required for the protein to function in vitro (Zamore et al. 1992), but the apparent $\mathrm{U}_{2} \mathrm{AF}^{65}$ homolog from Drosophila, which is essential for viability (Kanaar et al. 1993), does not require its RS region to provide its essential function (D. Rio, pers. comm.). This difference in RS domain requirement between fly U2AF and chicken ASF/SF2 could reflect any of several possibilities, one being a species-specific difference in the function of RS domains. However, it may also be that the RS regions in the two proteins are not functionally equivalent. The $U 2 \mathrm{AF}^{65}$ region has fewer consecutive RS dipeptides and also contains RD and RE dipeptides, which are not characteristic of SR protein RS domains. Further, although there is considerable evidence that the function of RS domains in SR proteins involves protein-protein interactions (e.g., Wu and Maniatis 1993; Kohtz et al. 1994|, it has been suggested that the function of the U2AF RS region may be to facilitate RNA-RNA interactions (e.g., between U2 snRNA and the branch site; Lee et al. 1993). However, whatever the basis for the difference between $\mathrm{U}_{2} \mathrm{AF}^{65}$ and ASF/SF2, our results have established that the ASF/ SF2 RS domain is essential for the viability of cultured DT40 cells. It should be possible in the future to obtain more insights into the role of RS domains by testing the ability of heterologous RS domains (e.g., from U2AF ${ }^{65}$ and other SR proteins) to substitute for the ASF/SF2 RS domain.

The system for gene disruption we have described here offers the potential for genetic analysis of essential vertebrate genes involved in a variety of basic cellular processes. We imagine it will be particularly useful for studying genes that lack a clear yeast counterpart, or which are suspected of playing a more complex role in metazoans than in yeast. Of course, genes that function in specific developmental or tissue-specific pathways will continue to require whole-organism systems for full analysis. Indeed, it could be that ASF/SF2 has developmental or tissue-specific functions that will be revealed by studies employing, for example, transgenic mice. However, for genes that function in basic cellular processes, such as splicing, the system we have developed is relatively simple and allows several genetic manipulations not feasible previously with cultured cells. We believe that it will prove useful for analysis of a variety of essential genes.

\section{Materials and methods \\ Cloning of chicken ASF/SF2 gene}

A chicken spleen cDNA library $\left(\sim 5 \times 10^{5}\right.$ plaques; Clonetech $)$ was screened using human ASF/SF2 cDNA (Ge et al. 1991) as probe. Five positive plaques were isolated and purified. cDNA inserts were excised by EcoRI digestion, cloned into pBluescript (Stratagene) and sequenced. Double-stranded DNA sequencing was performed as manufacturer's description (U.S. Biochemical). Chicken ASF/SF2 cDNA was then used to screen a chicken genomic library (Stratagene). From $1 \times 10^{6}$ plaques, two positive clones were obtained. Phage DNAs were purified and digested with SmaI. Fragments of the digestions were inserted into the EcoRV site of pBluescript. A total of $8 \mathrm{~kb}$ covering the entire chicken ASF/SF2 gene was sequenced.

\section{Plasmid constructs}

All plasmids used in this study were constructed by standard subcloning procedures. In brief, neomycin and hygromycin-resistance genes (Buerstedde and Takeda 1991; Takata et al. 1994), which are under control of the chicken $\beta$-actin promoter, were inserted in the StyI site in the first exon of chicken ASF/SF2 gene by blunt-end ligation, giving the constructs Neo-ASF/SF2 and Hygro-ASF/SF2. To construct the ASF/SF2 expression vector Puro-ASF, flu-hASF/SF2 cDNA fragment was excised from pCF21 (Wang and Manley 1995) by BamHI and inserted into the $E c o R I$ site, which lies downstream of chicken $\beta$-actin promotor, of the PA-puro vector containing the puromycin-resistance gene (Takata et al. 1994). hASF/SF2 cDNA in Puro-ASF was replaced by SRp40 (Screaton et al. 1995), SC35 (Fu and Maniatis 1992b), Gly, NR-d, MR-g, RS-b, RS-c, or $\Delta$ RS (Zuo and Manley 1993) cDNAs, giving the corresponding expression vectors used in complementation assays. To construct the vector tTA, hASF/ SF2 cDNA in Puro-ASF was replaced with tetR-VP16 cDNA (Gossen and Bujard 1992). Flu-hASF cDNA was put under the control of the tetO-minimal promoter (Gossen and Bujard 1992) and a histidinol-resistance gene (Takata et al. 1994) was inserted into the same plasmid, giving the vector $\mathrm{mASF} / \mathrm{SF} 2$. Details of constructions are available upon request.

\section{Cell culture and transfections}

DT40 cells were maintained in RPMI 1640 medium, supplemented with $10 \%$ fetal bovine serum (HyClone) and $1 \%$ chicken serum (Sigma) at $37^{\circ} \mathrm{C}$ and $5 \% \mathrm{CO}_{2}$. Cells were routinely split to $1 \times 10^{5}$ per ml every other day. For each transfection, $10^{7}$ cells were suspended in $0.5-\mathrm{ml}$ PBS containing 30 $\mu \mathrm{g}$ linearized plasmid and electroporated with a Gene Pulser apparatus (BioRad) at $550 \mathrm{~V}$ and $25 \mu \mathrm{F}$. Following electroporation, cells were transfered to $20 \mathrm{ml}$ fresh medium lacking drugs 
and incubated for $24 \mathrm{hr}$. Cells were then resuspended in $90 \mathrm{ml}$ medium containing the appropriate drugs and divided into four 96-well plates (Falcon). After 7-10 days, drug-resistant colonies were transfered to 24-well plates. Concentrations of drugs used in this study were: $2 \mathrm{mg} / \mathrm{ml} \mathrm{G} 418$ (GIBCO-BRL), $1.5 \mathrm{mg} / \mathrm{ml}$ hygromycin (Calbiochem), $0.5 \mu \mathrm{g} / \mathrm{ml}$ puromycin (Sigma), $1 \mathrm{mg} /$ $\mathrm{ml}$ histidinol (Sigma), and $1 \mu \mathrm{g} / \mathrm{ml}$ tetracycline hydrochloride (Sigma). Cells containing the tTA expression vector, which expresses tetR-VP16, grew slower than control cells. None of the other vectors used had a significant effect on growth.

\section{DNA and RNA analysis}

Genomic DNAs were isolated as described (Sambrook et al. 1989). Probes were obtained by restriction digestion of chicken ASF/SF2 genomic DNA and fragment purification, and labeled with $\alpha-{ }^{32}$ P-dATP by random priming. Southern blotting was performed as described (Sambrook et al. 1989). Total RNA samples were isolated from $10^{8}$ cells as described previously (Chomczynski and Sacchi 1987). Concentrations of RNAs were determined by UV absorption and formaldehyde agarose gel electrophoresis. A plasmid containing the first exon and part of the first intron of $c A S F / S F 2$ was linearized by $B s p E I$ digestion for in vitro transcription in the presence of $\alpha^{-32} \mathrm{P}-\mathrm{GTP}$. Twenty micrograms of each RNA sample and 200 cps probe were hybridized at $42^{\circ} \mathrm{C}$ overnight. RNase digestions with RNase $\mathrm{A}$ and $\mathrm{T} 1$ were performed as described (Sambrook et al. 1989). Protected fragments were separated on $5 \%$ polyacrylamide- $8 \mathrm{~m}$ urea gels and detected by autoradiography.

\section{Western blotting analysis}

Cells $\left(\sim 2 \times 10^{6}\right)$ were harvested, washed with PBS and lysed in $60 \mu \mathrm{l} 1 \times$ Laemmli loading buffer. Following brief sonication and boiling, aliquots (routinely 50\%) were subjected to $10 \%$ SDS-PAGE. After transfer to nitrocellulose membrane, proteins were detected by antiflu mAB 12CA5 (Field et al. 1988) using the ECL method (Amersham).

\section{Preparation and analysis of ${ }^{3} \mathrm{H}$-labeled RNA}

\#3 cells were grown in the absence of tet for $24 \mathrm{hr}$ or in the presence of tet for 24 or $48 \mathrm{hr}$. After adjusting the cell concentration to $10^{6}$ cells per $\mathrm{ml}$, four $\mathrm{mls}$ of each suspension were labeled with $50 \mu \mathrm{Ci} / \mathrm{ml}{ }^{3} \mathrm{H}$-uridine for $30 \mathrm{~min}$. Total RNAs were isolated and subjected to two rounds of selection on oligo/dT) cellulose as described (Sambrook et al. 1989). Radioactivity of total and poly $(\mathrm{A})^{+}$RNAs were quantitated by scintillation counting. $2.5 \times 10^{4} \mathrm{cpm}$ of each sample was resolved on $0.9 \%$ formaldehyde agarose gel. Following electrophoresis, the gel was soaked in $\mathrm{EN}^{3} \mathrm{HANCE}$ (DuPont) for $3 \mathrm{hr}$ and then in cold water for $1 \mathrm{hr}$. The dried gel was exposed to X-ray film at $-80^{\circ} \mathrm{C}$ for five days, and the film was then scanned with a densitometer (Bioimage, Millipore).

\section{Acknowledgments}

We thank Tomohiro Kurosaki for providing DT40 cells, drug resistance genes, and the PA-puro vector; Roland Tacke for providing SRp40 cDNA; Yan Chen for DNA sequencing; James Shanley for computer analysis and help in preparing the figures; and Maria Riley for assistance in preparing the manuscript. We also thank lab members for helpful discussions and comments. This work was supported by National Institutes of Health grant GM 48529.

The publication costs of this article were defrayed in part by payment of page charges. This article must therefore be hereby marked "advertisement" in accordance with 18 USC section 1734 solely to indicate this fact.

\section{References}

Ben-David, Y., M.R. Bani, B. Chabot, A. De Koven, and A. Bernstein. 1992. Retroviral insertions downstream of the hetergeneous nuclear ribonucleoprotein Al gene in erythroleukemia cells: Evidence that Al is not essential for cell growth. Mol. Cell. Biol. 12: 4449-4455.

Buerstedde, J.-M. and S. Takeda. 1991. Increased ratio of targeted to random integration after transfection of chicken $B$ cell lines. Cell 67: 179-188.

Cáceres, J.F. and A.R. Krainer. 1993. Functional analysis of premRNA splicing factor SF2/ASF structural domains. EMBO J. 12: 4715-4726.

Cáceres, J.F., S. Stamm, D.M. Helfman, and A.R. Krainer. 1994. Regulation of alternative splicing in vivo by overexpression of antagonistic splicing factors. Science 265: 1706-1709.

Cavaloc, Y., M. Popielarz, J.P. Fuchs, R. Gattoni, and J. Stevenin. 1994. Characterization and cloning of the human splicing factor 9G8: A novel $35 \mathrm{kDa}$ factor of serine/arginine protein family. EMBO J. 13: 2639-2649.

Chomczynski, P. and N. Sacchi. 1987. Single-step method isolation by acidic guanidinium thiocyanate-phenol-chloroform extraction. Anal. Biochem. 162: 156-159.

Crispino, J.D., B.J. Blencowe, and P.A. Sharp. 1994. Complementation by SR proteins of pre-mRNA splicing reactions depleted of U1 snRNP. Science 265: 1866-1869.

Eperon, I.C., D.C. Ireland, R.A. Smith, A. Mayeda, and A.R. Krainer. 1993. Pathways for selection of 5 ' splice sites by U1 snRNPs and SF2/ASF. EMBO J. 12: 3607-3617.

Field, J., J.I-. Nikawa, D. Broek, B. MacDonald, L. Rodgers, I.A. Wilson, R. Lerner, and M. Wigler. 1988. Purification of a RAS-responsive adenyl cyclase complex from Saccharomyces cerevisiae by use of an epitope addition method. Mol. Cell. Biol. 8: 2159- 2165.

Fu, X.-D. 1993. Specific commitment of different pre-mRNAs to splicing by single SR proteins. Nature 365: $82-85$.

. 1995. The superfamily of arginine/serine-rich splicing factors. RNA 1: 663- 680 .

Fu, X.-D. and T. Maniatis. 1990. Factor required for mammalian spliceosome assembly is localized to discrete regions in the nucleus. Nature 343: 437-441.

- 1992a. The 35-kDa mammalian splicing factor mediates specific interactions between $\mathrm{U} 1$ and $\mathrm{U} 2$ small nuclear ribonucleoprotein particles at the 3' splice site. Proc. Natl. Acad. Sci. 89: 1725-1729.

- 1992b. Isolation of a complementary DNA that encodes the mammalian splicing factor SC35. Science 256: 535-538.

Fu, X.-D., A. Mayeda, T. Maniatis, and A.R. Krainer. 1992. General splicing factors SF2 and SC35 have eqivalent activities in vitro, and both affect alternative $5^{\prime}$ and $3^{\prime}$ splice site selection. Proc. Natl. Acad. Sci. 89: 11224-11228.

Ge, H. and J.L. Manley. 1990. A protein factor, ASF, controls cell-specific alternative splicing of SV40 early pre-mRNA in vitro. Cell 62: 25-34.

Ge, H., P. Zuo, and J.L. Manley. 1991. Primary structure of the human splicing factor ASF reveals similarities with Drosophila regulators. Cell 66: 373-382.

Gossen, M. and H. Bujard. 1992. Tight control of gene expression in mammalian cells by tetracycline-responsive promoters. Proc. Nat1. Acad. Sci. 89: 5547-5551.

Haynes, S.R., G. Raychaudhuri, and A.L. Beyer. 1990. The Drosophila Hrb98DE locus encodes four protein isoforms homologous to the A1 protein of mammalian heterogeneous nuclear ribonucleoprotein complexes. Mol. Cell. Biol. 10: $316-323$.

Hoshijima, K., K. Inoue, I. Higuchi, H. Sakamoto, and Y. 
Shimura. 1991. Control of doublesex alternative splicing by transformer and transformer-2 in Drosophila. Science 252: 833-836.

Jamison, S.F., Z. Pasman, J. Wang, C. Will, R. Lührmann, J.L. Manley, and M.A. Garcia-Blanco. 1995. U1 snRNP-ASF/SF2 interaction and $5^{\prime}$ splice site recognition: Characterization of required elements. Nucleic Acids Res. 23: 3260-3267.

Kanaar, R., S.E. Roche, E.L. Beall, M.R. Green, and D.C. Rio. 1993. The conserved pre-mRNA splicing factor U2AF from Drosophila: Requirement for viability. Science 262: 569 573.

Kanopka A., O. Mühlemann and G. Akusjärvi. 1996. SR proteins that are essential for generic pre-mRNA splicing inhibit splicing of a regulated adenovirus pre-mRNA. Nature 381: 535-538.

Kelley, R.L. 1993. Initial organization of the Drosophila dorsoventral axis depends on an RNA-binding protein encoded by the squid gene. Genes \& Dev. 7: 948-960.

Kim, Y.-J., P. Zuo, J.L. Manley, and B.S. Baker. 1992. The Drosophila RNA-binding protein RBP1 is localized to transcriptionally active sites of chromosomes and shows a functional similarity to human splicing factor ASF/SF2. Genes \& Dev. 6: 2569-2579.

Kohtz, J.D., S.F. Jamison, C.L. Will, P. Zuo, R. Lührmann, M.A. Garcia-Blanco, and J.L. Manley. 1994. Protein-protein interactions and 5 '-splice-site recognition in mammalian mRNA precursors. Nature 368: 119-124.

Krainer, A.R., G.C. Conway, and D. Kozak. 1990a. The essential pre-mRNA splicing factor SF2 influences 5' splice site selection by activating proximal sites. Cell 62: 35-42.

1990b. Purification and characterization of pre-mRNA splicing factor SF2 from Hela cells. Genes \& Dev. 4: 11581171.

Krainer, A.R., A. Mayeda, D. Kozak, and G. Binns. 1991. Functional expression of cloned human splicing factor SF2: Homology to RNA-binding proteins, Ul $70 \mathrm{k}$, and Drosophila splicing regulators. Cell 66: 383-394.

Kraus, M.E. and J.T. Lis. 1994. The concentration of B52, an essential splicing factor and regulator of splice site choice, is critical for Drosophila development. Mol. Cell. Biol. 14: $5360-5370$.

Lee, C.G., P.D. Zamore, M.R. Green, and J. Hurwitz. 1993. RNA annealing activity is intrinsically associated with U2AF. $I$. Biol. Chem. 268: 13472-13478.

Li, Y. and J.B. Dodgson. 1995. The chicken $H M G-17$ is dispensable for cell growth in vitro. Mol. Cell. Biol. 15: 5516-5523.

McNally L.M. and M.T. McNally. 1996. SR protein splicing factors interact with the Rous sarcoma virus negative regulator of splicing element. J. Virol. 70: 1163-1172.

Manley, J.L. and R. Tacke. 1996. SR proteins and splicing control. Genes \& Dev. 10: 1569-1579.

Mattox, W., L. Ryner, and B.S. Baker. 1992. Autoregulation and multifunctionality among trans-acting factors that regulate alternative pre-mRNA processing. J. Biol. Chem. 267: 19023-19026.

Matunis, E.L., M.J. Matunis, and G. Dreyfuss. 1992. Characterization of the major hnRNP proteins from Drosophila melanogaster. J. Cell Biol. 116: 257-269.

Mayeda, A. and A.R. Krainer. 1992. Regulation of alternative pre-mRNA splicing by hnRNP A1 and splicing factor SF2. Cell 68: 365-375.

Moore, M.J., C.C. Query, and P.A. Sharp. 1993. Splicing of precursors to mRNAs by the spliceosome. In RNA world. (ed. R. Gesteland and J. Atkin) pp. 303-357. Cold Spring Harbor Laboratory Press, Cold Spring Harbor, NY.

Peng, X. and S.M. Mount. 1995. Genetic enhancement of RNA- processing defects by a dominant mutation in B52, the Drosophila gene for an SR protein splicing factor. Mol. Cell. Biol. 15: 6273-6282.

Ramachatesingh, J., A.M. Zahler, K.M. Neugebauer, M.B. Roth, and T.A. Cooper. 1995. A subset of SR proteins activates splicing of the cardiac troponin $\mathrm{T}$ alternative exon by direct interactions with an exonic enhancer. Mol. Cell. Biol. 15: 4898-4907.

Ring, H.Z. and J.T. Lis. 1994. The SR protein B52/SRp55 is essential for Drosophila development. Mol. Cell. Biol. 14: 7499-7506.

Roscigno, R.F. and M.A. Garcia-Blanco. 1995. SR proteins escort U4/6.U5 tri-snRNP to the spliceosome. RNA 1: 692-706.

Sambrook, J., E.F. Fritsch, and T. Maniatis. 1989. Molecular cloning: A laboratory manual. 2nd ed. Cold Spring Harbor Laboratory Press, Cold Spring Harbor, NY.

Screaton, G.R., J.F. Cáceres, A. Mayeda, M.V. Bell, M. Plebanski, D.G. Jackson, J.I. Bell, and A.R. Krainer. 1995. Identification and characterization of three members of the human SR family of pre-mRNA splicing factors. EMBO $J$. 14: 43364349.

Spector, D.L. 1993. Macromolecular domains within the cell nucleus. Annu. Rev. Cell Biol. 9: 265-315.

Staknis, D. and R. Reed. 1994. SR proteins promote the first specific recognition of pre-mRNA and are present together with the Ul small nuclear ribonucleoprotein particle in a general splicing enhancer complex. Mol. Cell. Biol. 14: 7670-7682.

Sun, Q., A. Mayeda, R.K. Hampson, A.R. Krainer, and F.M. Rottman. 1993. General splicing factor SF2/ASF promotes alternative splicing by binding to an exonic splicing enhancer. Genes \& Dev. 7: 2598-2608.

Tacke, R. and J.L. Manley. 1995. The human splicing factors ASF/SF2 and SC35 possess different, functionally significant RNA binding specificities. EMBO J. 14: 3540-3551.

Takata, M., H. Sabe, A. Hata, T. Inazu, Y. Homma, T. Nukada, H. Yamamura, and T. Kurosaki. 1994. Tyrosine kinases Lyn and Syk regulate $\mathrm{B}$ cell receptor-coupled $\mathrm{Ca}^{2+}$ mobilization through distinct pathways. EMBO J. 13: 1341-1349.

Takeda, S., E.L. Masteller, C.B. Thompson, and J.-M. Buerstedde. 1992. RAG-2 expression is not essential for chicken immunoglobulin gene conversion. Proc. Natl. Acad. Sci. 89: 4023-4027.

Tarn, W.Y. and J.A. Steitz. 1994. SR proteins can compensate for the loss of Ul snRNP functions in vitro. Genes \& Dev. 8: 2704-2717.

- 1995. Modulation of $5^{\prime}$ splice site choice in pre-mRNA by two distinct steps. Proc. Natl. Acad. Sci. 92: 2504-2508.

Tian, M. and T. Maniatis. 1992. Positive control of pre-mRNA splicing in vitro. Science 256: 237-240.

. 1993. A splicing enhancer complex controls alternative splicing of doublesex pre-mRNA. Cell 74: 105-114.

Vellard, M., A. Sureau, J. Soret, C. Martinerie, and B. Perbal. 1992. A potential splicing factor is encoded by the opposite strand of the trans-spliced c-myb exon. Proc. Natl. Acad. Sci. 89: 2511-2515.

Wang, J. and J.L. Manley. 1995. Overexpression of the SR proteins ASF/SF2 and SC35 influences alternative splicing in vivo in diverse ways. RNA 1: 335-346.

Wang, Z., H.M. Hoffmann, and P.J. Grabowski. 1995. Intrinsic U2AF binding is modulated by exon enhancer signals in parallel with changes in splicing activity. RNA 1: 21-35.

Watakabe, A., K. Tanaka, and Y. Shimura. 1993. The role of exon sequences in splice site selection. Genes \& Dev. 7: 407-418.

Woppmann, A., C.L. Will, U. Kornstadt, P. Zuo, J.L. Manley, 
and R. Lührmann. 1993. Identification of an snRNP-associated kinase activity that phosphorylates arginine/serine rich domains typical of splicing factors. Nucleic Acids Res. 21: 2815-2822.

Wu, J.Y. and T. Maniatis. 1993. Specific interactions between proteins implicated in splice site selection and regulated alternative splicing. Cell 75: 1061-1070.

Yang, X., M.R. Bani, S.J. Lu, S. Rowan, Y. Ben-David, and B. Chabot. 1994. The Al and AlB proteins of heterogeneous nuclear ribonucleoparticles modulate $5^{\prime}$ splice site selection in vivo. Proc. Natl. Acad. Sci. 91: 6924-6928.

Zahler, A.M. and M.B. Roth. 1995. Distinct functions of SR proteins in recruitment of Ul small nuclear ribonucleoprotein to alternative $5^{\prime}$ splice sites. Proc. Natl. Acad. Sci. 92: 2642-2646.

Zahler, A.M., W.S. Lane, J.A. Stolk, and M.B. Roth. 1992. SR proteins: A conserved family of pre-mRNA splicing factors. Genes \& Dev. 6: 837-847.

Zahler, A.M., K.M. Neugebauer, W.S. Lane, and M.B. Roth. 1993. Distinct functions of SR proteins in alternative premRNA splicing. Science 260: 219-222.

Zamore, P.D. and M.R. Green. 1989. Identification, purification, and characterization of U2 small nuclear ribonucleoprotein auxiliary factor. Proc. Natl. Acad. Sci. 86: 9243 9247.

Zamore, P.D., J.G. Patton, and M.R. Green. 1992. Cloning and domain structure of the mammalian splicing factor U2AF. Nature 355: 609-614.

Zuo P. and T. Maniatis. 1996. The splicing factor U2AF ${ }^{35}$ mediates critical protein-protein interactions in constitutive and enhancer-dependent splicing. Genes \& Dev. 10: 13561368.

Zuo, P. and J.L. Manley. 1993. Functional domains of the human splicing factor ASF/SF2. EMBO J. 12: 4727-4737.

. 1994. Human splicing factor ASF/SF2 can specifically recognize pre-mRNA 5' splice sites. Proc. Natl. Acad. Sci. 91: 3363-3367. 


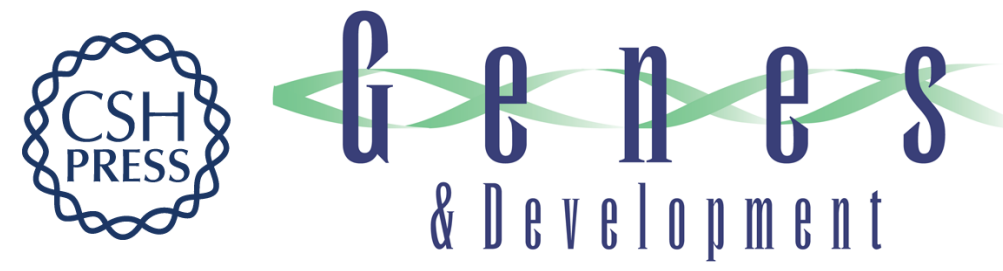

\title{
Targeted disruption of an essential vertebrate gene: ASF/SF2 is required for cell viability.
}

\author{
J Wang, Y Takagaki and J L Manley
}

Genes Dev. 1996, 10:

Access the most recent version at doi:10.1101/gad.10.20.2588

$\begin{array}{ll}\text { References } & \begin{array}{l}\text { This article cites } 67 \text { articles, } 42 \text { of which can be accessed free at: } \\ \text { http://genesdev.cshlp.org/content/10/20/2588.full.html\#ref-list-1 }\end{array}\end{array}$

License

Email Alerting Receive free email alerts when new articles cite this article - sign up in the box at the top Service right corner of the article or click here.

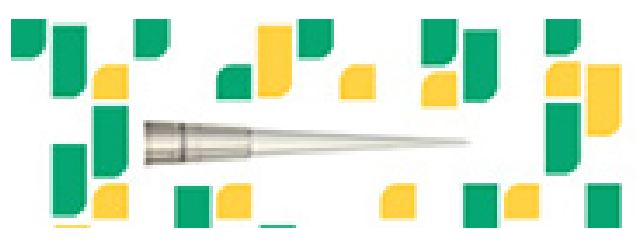

Focused on your science. 